\title{
Haptic virtualization of surfaces: feeling textiles on your phone
}

\author{
Tung Le, Christopher Martin, Annerose Braune* \\ Chair of Automation Engineering, Technische Universität Dresden, Germany \\ *Corresponding author E-mail address: annerose.braune@tu-dresden.de
}

\section{INFO}

CDAPT, ISSN 2701-939X

Peer reviewed article

2021, Vol. 2, No. 1, pp. 80-90

DOI 10.25367/cdatp.2021.2.p80-90

Received: 08 June 2021

Accepted: 07 July 2021

Available online: 13 July 2021

\begin{abstract}
The haptic impression of textile surface properties has a decisive influence on its evaluation and ultimately on its acceptance and usability. Many solutions are used to replicate a static contour or shape, e.g. to feel controls on common touch displays. In contrast, this project investigates whether it is possible to simulate the roughness or friction behavior of a textile surface using a commercially available mobile device.
\end{abstract}

\author{
Keywords \\ Haptic human machine interfaces, \\ Textile surface, \\ Roughness, \\ Mobile consumer devices, \\ Tactile rendering
}

(C) 2021 The authors. Published by CDAPT.

This is an open access article under the CC BY-NC-ND license https://creativecommons.org/licenses/ peer-review under responsibility of the scientific committee of the CDAPT.

\section{Introduction}

The importance of virtual reality applications continuously increases e.g. in the field of online shopping. Yet, potential buyers have not been able to perceive product properties throughout the haptic modality, e.g. in textile industries. Humans have haptic sensors to feel the properties when swiping over the textiles. In case of virtual reality, however, humans do not interact with the real textile, but with a device. Now this device must represent the textile and has to simulate textile properties as far as possible in such a way that realistic sensations are generated.

In several research projects, devices have already been developed that simulate textile properties [1,2]. The reproduction of static contours and shapes is already being used, for example, to feel control elements on touch displays [3]. These shapes represent static elements as virtual elevations on a flat screen. However, if you want to feel the texture, the roughness or the friction behavior of a textile surface by stroking your fingertip over it, the contours to be simulated are microscopically small. In this article, we will now investigate whether normal commercial touch displays in mobile devices can be used for this purpose. 
Typical mobile devices have features for vibration alarms. They act as an alternative signal transmitter if the acoustic signal is perceived as disturbing during a call or the visual display cannot be perceived immediately. For the user it is only necessary to distinguish certain vibration patterns from each other or to perceive a vibration haptically at all. The engines of the mobile phones are not designed for a large variation of vibration intensities and therefore only a low resolution of the intensity exists. However, due to the widespread use and the easy accessibility of smartphones and tablets, the "misappropriation" for textile surfaces will be discussed.

The following chapter 2 therefore first describes the human sensors for haptic perception on the finger and their essential capabilities. Chapter 3 presents existing haptic devices for user interfaces both in terms of the haptic properties to be displayed and from the aspect of accessibility. Chapters 4 and 5 subsequently describe the developed mobile application and a user study conducted with it. Chapter 6 evaluates the results and derives conclusions about usability.

\section{Human haptic perception}

When evaluating the importance of each human sensory organ, one approach is to analyze the number of receptors and the amount of information perceived. Whereas most information is processed through the visual channel, the human skin comes out as the second most important sensory organ, being about 1000 times larger than the other ones [4]. The skin's sensors are distributed over the entire human body and the receptor density depends on the part of the body. For example, the fingers can detect extremely fine structures (down to $2.5 \mathrm{~mm}[5,6]$ ), while at the back, the resolving capacity is lower, so that structures can no longer be distinguished that well [5].

Haptic perception is described as all sensory and motoric functions that are responsible for the sense of touch (tactile perception) and movement (kinaesthetic perception). While tactile perception is perceived through the skin's mechanoreceptors, kinaesthesia describes activity and perception of the muscles, tendons, and joints [7]. Haptic perception describes the process of actively exploring objects and surfaces including both perception receptors (Fig. 1). Important tactile receptors are

- Merkel cells: Slowly Adapting type 1 (SA-I)

- Ruffini corpuscles: Slowly Adapting type 2 (SA-II)

- Meissner's corpuscles: Rapidly Adapting (RA) resp. Fast Adapting type 1 (FA-I)

- Pacinian corpuscles: Fast Adapting type 2 (FA-II)

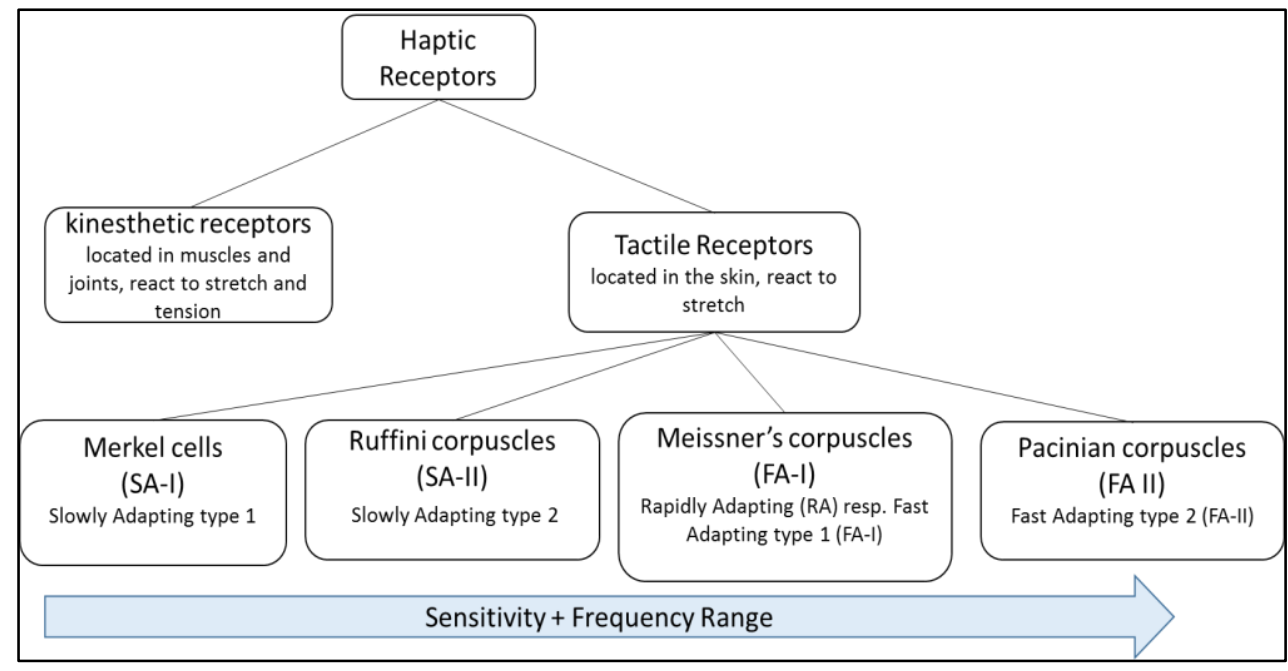

Figure 1: Classification of haptic sensors, following [7]

The slowly adapting receptors (SA-I and SA-II) detect the strength of the deformation, i.e. the depth of the skin's stretching. They are mainly responsible for perceiving more static deformation [5]. Fig. 2 summarizes 
the tactile perception by displaying the frequency ranges which each tactile sensor responds to. Furthermore, the figure provides the absolute threshold area for each receptor type. The average displacement threshold for the SA receptors is detected at about $50 \mu \mathrm{m}$ (dashed line in Fig. 2) and they have their highest sensitivity at less than 20 changes per second $(10 \mathrm{~Hz})$. The rapidly adapting sensors (RA and FA) adapt very quickly to changing deformations. However, their sensitivity to stimuli decreases again after a short time. They therefore tend to detect rapidly changing stimulus patterns and are thus used to detect relative movements of objects on the skin and mechanical vibratory deformation. These sensors can sense vibrations at around 200 to $300 \mathrm{~Hz}$ with an amplitude starting from $10 \mu \mathrm{m}$. This is an indicator for the higher degree of sensitivity for the RA resp. FA sensors.

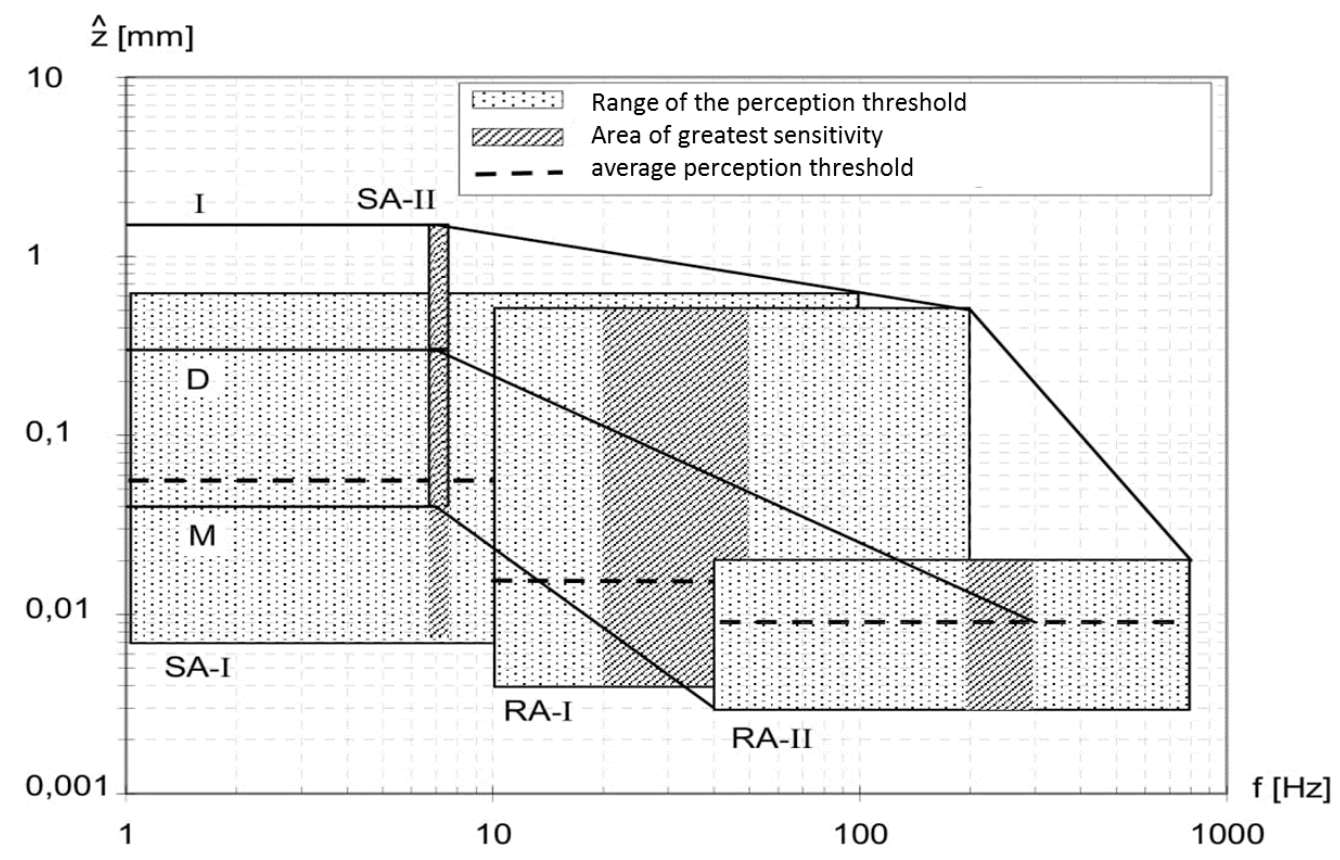

Fig. 2: Frequency dependent perception thresholds of tactile receptors [27]

The neurophysiological point of view is not ideally applicable to the human perception. Sensory stimuli may be filtered, but also combined for a more sensitive perception. Psychophysics, as in [6], discusses the effects of physical stimuli on the subjective sensation and perception. The detection threshold for a non-moving fingertip, e.g., is about $1 \mathrm{~mm}$ [8], whereas active haptic perception can detect bumps down to $0.85 \mu \mathrm{m}$ [9]. This allows distinguishing the roughness level of textile surfaces for evaluating its comfort.

\section{Haptic Devices}

With the biological concepts of haptic perception in mind, devices can be designed to target the sensor types as described in the previous chapter. According to Fig. 1, haptic devices can be divided into those that stimulate kinaesthetic receptors and those that stimulate tactile receptors. Kinaesthetic devices stimulate the receptors in muscles, tendons and joints. They use the feedback of force to interact with users. Such force feedback devices are often used as virtual training simulation cockpits or robotic assisted teleoperations [10]. But in this paper, we restrict the applicable domain to textile surfaces. Those are primarily sensed by skin receptors, which leads to an in-depth look for tactile devices.

\subsection{Concepts of Tactile Devices}

For contact-based mechanical stimulation there are two common approaches. The first one can be described as static displacement. Such devices replicate the shape and surface of an object as close-toreality as possible. The virtualization process requires multiple actor pins arranged in a matrix (pin-array). By giving each pin a separately controllable displacement output, different surface forms can be applied onto the device. This approach can be achieved, for example, piezo-electrically [11] or with electro- or 
magnetorheological fluids [12]. A high spatial resolution array, with pins being about $0.5 \mathrm{~mm}$ apart from each other, was accomplished by using thermal-sensitive hydrogels [13].

The second approach can be described as vibrotactile. These devices do not aim for replicating the static shape of the surface itself. Instead they focus on giving a realistic impression and sensation of haptic surface properties, when feeling a virtualized object. This approach is supported by means of vibration $[5,7]$ to stimulate FA receptors (cf. Chapter 2). Vibrotactile output of these devices is rendered through position tracking of the human contact area, such as the fingertip. Although not necessarily required, this method can be used in conjunction with previously described pin-arrays (e.g. [14-16]). Adding vibrotactile elements to pin-arrays vastly improves user experience [17].

More recent advances for tactile stimulation for consideration are non-contact stimulation via ultrasonic waves [18], generating friction with electrostatic fields [19] or electrotactile displays that directly induce current into the receptor's nerve endings [20]. These technologies are especially useful for low-noise devices over motoric actuators.

\subsection{Mobile consumer devices as haptic user interfaces}

Using previously described concepts, several haptic devices were developed, often as a result of research projects. These devices were not designed from the point of usability, but for very specific applications and further scientific studies. Their technical design usually requires additional equipment, making them therefore often quite large, heavy and thus uncomfortable for consumers and for transport. These research products are generally not easily accessible on the consumer market.

In contrast mobile consumer devices, such as smartphones and tablets, are prevalent around the world. Leading corporations are striving to equip common displays with tactile functionality. This enables feeling control elements such as buttons intuitively on displays through haptic feedback. The simulation of tactile surface properties creates significantly higher requirements.

Mobile devices utilize a vibrotactile approach (cf. Chapter 2) with two widespread technical implementations: eccentric rotating mass (ERM) and linear resonant actuator (LRA). ERM's rotating unbalance causes the device casing to vibrate on the rotational plane. Due to their simple nature, ERM technology is still more dominant on the mobile device market. However, if the haptic user experience is to be improved, manufacturers prefer newer LRA implementations. LRAs work similar to voice-coil principles of loudspeakers. Their dynamic properties are characterized by faster response times than ERM actuators [21]. LRAs are installed, e.g., in newer iPhone models with Apple's Taptic Engine [22] or in the Sony Xperia XZ2 device for enhancing media playback experience by additionally providing haptic feedback (Dynamic Vibration System - technology) [23].

\subsection{Conclusion}

The spatial pin densities of tactile pin arrays vary from $2 \mathrm{~mm}$ to $3 \mathrm{~mm}$ per pin (e.g. [12,14-16]), but can be reduced to $0.5 \mathrm{~mm}$ with manufacturing processes for semiconductors (cf. Section 3.1). When used as static displacement devices, this would be sufficient since the pin density meets the fingertips spatial threshold (cf. Chapter 2). However, textile surfaces generally have far more defined contours, where distances between surface levels are possibly within microns. In addition to a swiping movement, this results in a vibration from a finger's point of view. As human receptors can sense vibratory skin displacement up to $800 \mathrm{~Hz}$ (cf. Fig. 2), they can distinguish finer surface structures, such as textiles. Therefore, a vibrotactile approach is preferred over static displacement, to precisely stimulate FA receptors.

Since mobile devices, such as smartphones or tablets, are widely used, their suitability for a haptic user interface is the central task of this paper. Conventional built-in vibration motors oscillate at 7.000 to 12.000 rpm (rounds per minute) [7] and thus specifically target Pacinian corpuscles (cf. Chapter 2). Since they 
provide visual and auditive feedback, a multimodal aspect may be included, enhancing user experience by targeting multiple perception channels.

With these aspects in mind, we would like to test the following scenarios: Is it possible to feel the texture and roughness of the textile surface, the friction behavior and, to a certain extent, the softness of the textile surface when swiping over the display of a mobile device? Consequently, a mobile application is proposed, that is tested on Sony Xperia XZ3 mobile phone, the successor of the Sony Xperia XZ2 model on an Android operating system. With the integrated LRA, the haptic virtualization application substantially benefits from better transient response, as stated earlier.

\section{Mobile Application}

\subsection{Haptic rendering}

While the main purpose of mobile devices' vibrations is silent alerts, a haptic inspection process requires a precise control of the vibrating device. In this paper, the procedure of exploring a textile surface with a fingertip is virtualized by replacing the inspected surface with a smartphone's display. While the finger swipes over the touch screen, the application generates a vibration pattern according to the surfaces' contour, giving the impression of an actual textile by its property roughness.

In this section, we introduce an algorithm for continuous adaption of haptic feedback depending on the finger trajectory. For this purpose, the textile surfaces are measured and digitalized by 3D scans. The resulting point cloud serves as a function $z=f(x, y)$, with $x, y$ spanning the surface plain and $z$ being the corresponding height level (Fig. 3).

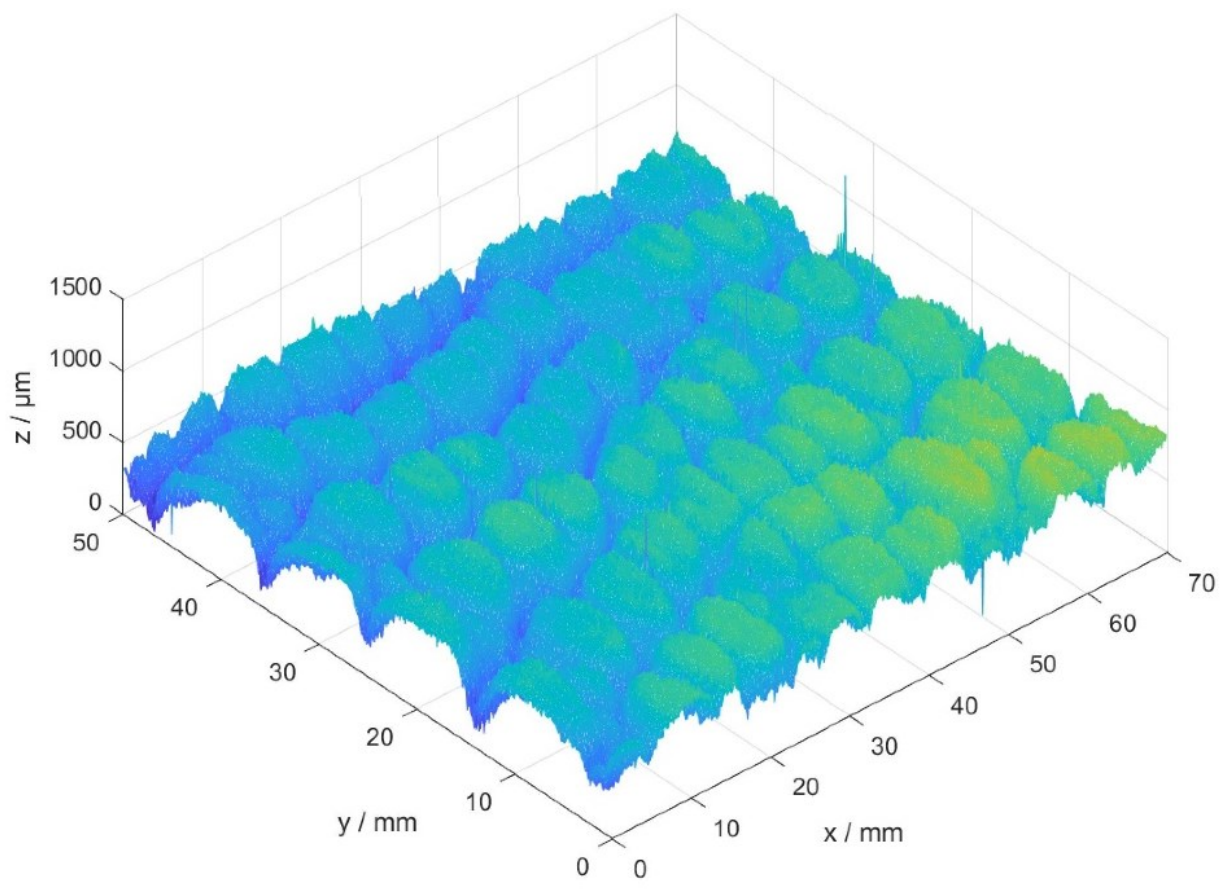

Fig. 3: 3D scan example of a textile surface

This function may be encoded within a grayscale image, with $z$ matching to the image's pixel values. Now, by tracking the finger's movement $\left[\begin{array}{lll}x(t) & y(t)\end{array}\right]^{T}$ and combining it with previously described height function results in a temporal height function:

$z(t)=f(x(t), y(t))$

Having the surface height variation history, this function is processed by a haptic feedback algorithm to set a proper output on the vibration element. Due to similarities in the auditory and haptic perception, the 
input data are pre-processed by means of Fourier transformation (Equation 2), a common approach implemented in haptic applications [14,24].

$F(\omega)=\mathcal{F}\{z(t)\}$

After performing Fourier analysis, a convenient amplitude must be applied onto the vibrating device. Allerkamp et al. [14] proposed a generalized haptic signal, consisting of two base frequencies at $40 \mathrm{~Hz}$ and $320 \mathrm{~Hz}$, thus targeting both SA and FA sensors (cf. Chapter 2). With a suitable superposition of the two frequencies, one tries to stimulate any haptic feeling. A similar basic idea underlies the RGB model. Here, too, any other color is generated by superposition of the 3 basic colors, i.e. by the superposition of the different wavelengths respectively the corresponding frequencies. However, our selected hardware in conjunction with its underlying vibration API [25] is limited to oscillate at a single pre-set frequency. As stated earlier, the concept is not to exactly replicate the surface contour, but to stimulate a comparable tactile impression. For this purpose, a weighted root mean square (RMS) is calculated from the discrete Fourier spectrum $F\left(\omega_{i}\right)$, as seen in Equation (3). An RMS emphasizes dominant frequencies over multiple minor frequencies and assesses them a higher value.

$P=c_{v} \sqrt{\frac{1}{n} \sum\left[c_{r e c}\left(\omega_{i}\right) \cdot F\left(\omega_{i}\right)\right]^{2}}$

$P$ describes the input parameter of Android's vibration API [25], representing the oscillation amplitude. Equation (3) includes a frequency-dependent weight factor $c_{r e c}$ that resembles different sensitivities of each sensor type (cf. Chapter 2). Values for $c_{r e c}$ are selected according to the relative average detection threshold (cf. Fig. 2Fig. 2). Consequently, sensors operating in higher frequency bands (FA-I, FA-II) are given a higher weight factor, as seen in Equation (4).

$c_{\text {rec }}(\omega)=\left\{\begin{array}{clc}1 / 6, & \text { for } & \omega<10 \mathrm{~Hz} \\ 1 / 2, & \text { for } & 10 \mathrm{~Hz} \leq \omega<40 \mathrm{~Hz} \\ 1, & \text { for } & 40 \mathrm{~Hz} \leq \omega<800 \mathrm{~Hz} \\ 0, & \text { else } & \end{array}\right.$

Before $P$ can finally be applied onto the vibration motor, Equation (3) still contains an unknown variable $c_{v}$. As the mobile device's displacement parameter are neither found in its data sheet, nor can it be reliably determined by internal sensory, this amplification constant will be discussed in a follow-up perception study (cf. Chapter 5).

\subsection{Android App}

Our approach was implemented by developing an app for Android-based handhelds. The app including the mobile device hardware serves as the haptic user interface, whereas textile-related information is stored on an external database. The app accesses the database by sending HTTP requests over the internet to its web service. The server provides a catalogue along with a property set (e.g. type of fabric, materials), containing textile surfaces being available for inspection. After browsing and on item selection, the app fetches the necessary data for tactile rendering from the web service, including the item's surface profile.

When developing an Android app, its interfaces (such as Wi-Fi, touchscreen, or sensors) can only be accessed throughout the operating system API that may cause limitations on the user interface. Inputwise, the retrieval of a finger's trajectory is controlled by the Android framework with efficiency purposes [26]. On the one hand, input data is delivered at undeterminable timestamps (being around $15 \mathrm{~ms}$ to $70 \mathrm{~ms}$ apart, depending on the rate of input change). On the other hand, multiple samples are batched together. Equation (2) from Chapter 4.1 is practically implemented by Fast Fourier Transformation (FFT). This algorithm though requires temporal equidistant sample data in contradiction to Android's motion data. In 
order to perform the FFT algorithm, additional samples are computed by means of linear interpolation (cf. Equation (5)), with $t_{i}, \vec{x}\left(t_{i}\right)$ being provided by the motion framework.

$\left(\begin{array}{l}x(t) \\ y(t)\end{array}\right)=\vec{x}(t)=\frac{\vec{x}\left(t_{i+1}\right)-\vec{x}\left(t_{i}\right)}{t_{i+1}-t_{i}}\left(t-t_{i}\right)+\vec{x}\left(t_{i}\right)$ with $t_{i} \leq t<t_{i+1}$

On the output-wise limitations it was discovered that sending a vibration request to the device's API causes the LRA module to halt, before oscillating with the desired amplitude. Too frequent updates seriously affect user experience. Therefore, a minimum update interval of $50 \mathrm{~ms}$ was found to feel continuous vibration and suppress perceivable stops of the actuator. A constant time interval is preferred, however, since a.) Android operating system does not provide real-time execution to guarantee specified cycle time, and b.) the algorithm could possibly miss on valuable input data. The time window for the FFT is equivalent to the current update interval and its time stamps have to be determined for each iteration.

\section{User study}

Finally, a user study is conducted for the mobile application. The study serves two purposes: subjective user evaluation and determining an amplification factor for $c_{v}$ (cf. Equation (3)).

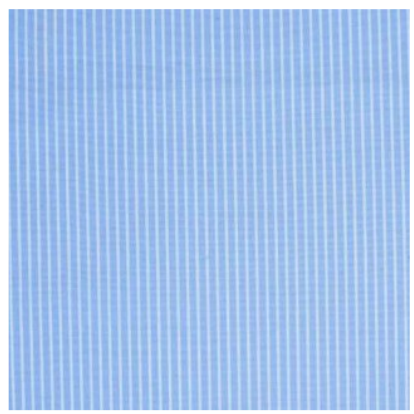

(a) H71 Shirting fabric, very smooth

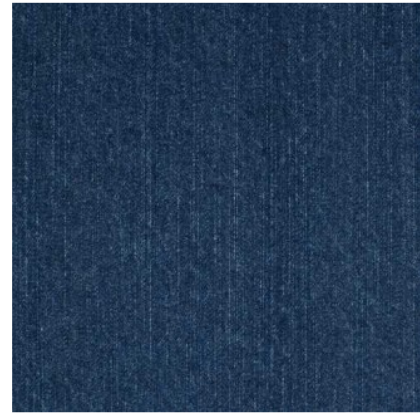

(b) B1 Denim, medium rough

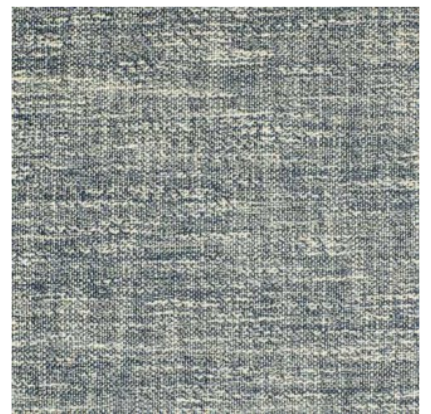

(c) H31 costume fabric, very rough

Fig. 4: Textile samples used in the user study with increasing roughness from left to right

The experimental setup consists of a Sony Xperia XZ3 smartphone and three textile surfaces with their structure ranging from very smooth to very rough (cf. Fig. 4). The subjects' task was to explore the textiles by moving their fingertip over the surface. For each surface their virtual resemblance on the smartphone shall be inspected and adjusted, so that the feeling of virtual and actual textile is as close as possible to the Point of Subjective Equality (PSE), e.g., introduced in [20]. For this purpose, a modified application was installed, which included a slider to modify the vibration intensity (cf. Fig. 5).

The subjects were asked to obtain a PSE value for a slow $(2 \mathrm{~cm} / \mathrm{s})$ and a fast $(10 \mathrm{~cm} / \mathrm{s})$ swiping movement, which were visually monitored. The smartphone was fixated on a table to suppress the device moving and to restrain receptor stimulation to the fingertip only.

13 subjects in total, six female and seven male, in the age from 18 to 34, resp. 50 and above participated in this experiment. Subjects were asked about their textile expertise, ranging from beginner to expert, where two declared as advanced and the remaining as beginners. None of them had experience in haptic human-machine interaction. Participants were given an introductory training phase with the total experiment duration lasting approx. 30 minutes. 

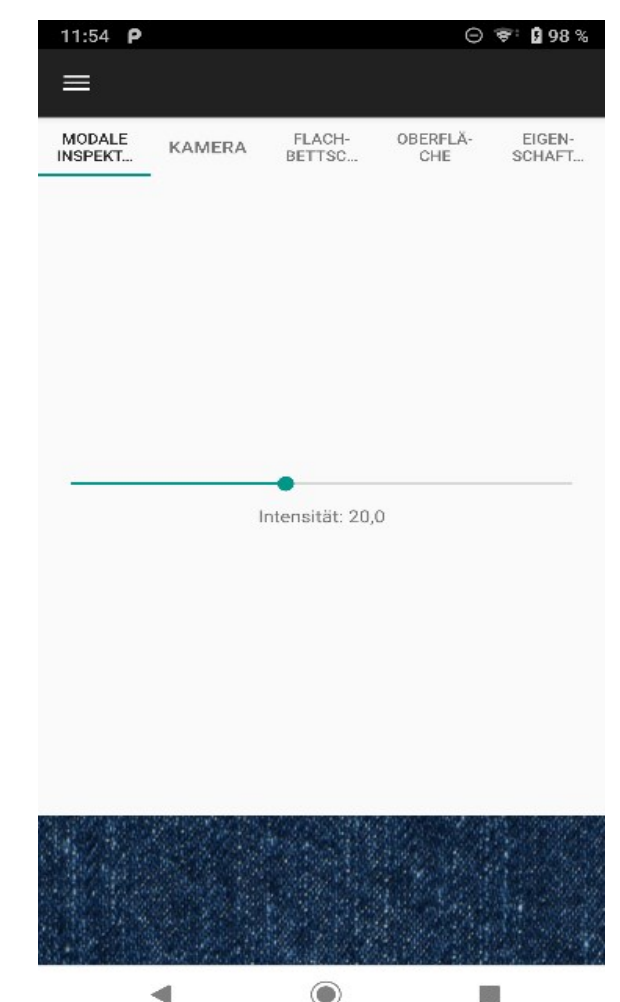

Fig. 5 : Textile inspection view of the user study's application

For each sub experiment the average PSE value resp. standard deviation from all participants are presented in Fig. 6. The medium rough textile B1 denim achieved the lowest relative standard deviation, meaning a high level of agreement from all subjects. B1's surface is mainly perceived by means of friction, rather than its structures and contours. The mobile device's oscillation direction parallel to the finger's motion proves to be advantageous over vertical displacement, when stimulating a friction effect. This result complies with the participants' comments: H71's shirting fabric surface was too smooth, when swiping over the real textile, complicating the replication on the handheld's glass surface. PSE values were set on the individual perception threshold, rather than identifying equal points of tactile feels. The H31 costume fabric on the other hand displayed strongly defined textures even in a static context, which cannot by imitated by the plain glass surface. Often the PSE values were set to maximum intensity, which exceeded the vibration's output range. Therefore, the mobile device as a haptic user interface may function with textiles within a specified roughness range, as surface virtualization for both too smooth and too rough surfaces collide with device limitations.
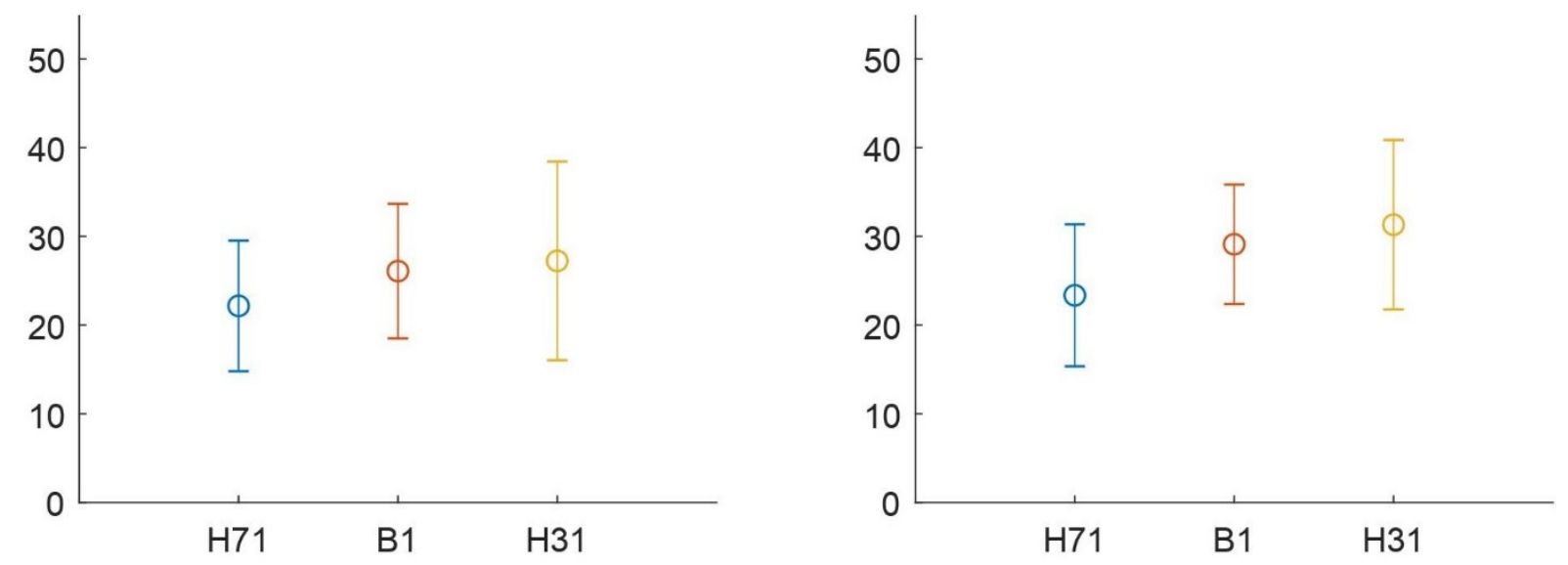

Fig. 6: Mean PSE values with standard deviations for slow movement (left) and fast movement (right) 
The algorithm described in Section 4.1 proves to be a suitable approach, but requires refinement, e.g. including textile properties (such as stiffness), since the PSE mean values show discrepancies among each sub-experiment while having the earlier discussed device restraints in mind.

The rather high standard deviations illustrate a highly individual perception, which requires a user-specific scaling factor. This could be determined by an initialization run with a few common reference textiles.

\section{Conclusions}

The aim of this project was to test whether properties of a textile surface such as roughness and friction behavior can be realistically simulated with the help of a mobile consumer device (cf. Section 3.2). For this purpose, it is helpful to be able to use measured and thus objectively detectable data.

In our case, the surfaces of the textiles were measured using a 3D scanner. From this, roughness parameters were determined according to DIN EN ISO 4287:2010-07 and serve as the basis for the haptic algorithm which controls the motor in the mobile device. Fig. 7 shows the output of the described haptic algorithm (x-axis) and the measured roughness values (y-axis). The plot shows the expected high degree of correlation between the considered quantities, since both characteristics were derived from the same measured value, the 3D scan. The correlation coefficients are 0.92 for the center roughness, 0.93 for the profile depth and 0.95 for the maximum roughness depth.

The results of the user study show that the algorithm described in Section 4.1 proves to be a suitable approach, but requires refinement, since the PSE mean values show discrepancies among each sub experiment. For a further improvement of the sensation, a consideration of additional objectively measurable parameters in the algorithm is also conceivable. For example, fiber stiffness is not yet taken into account by the 3D scan of the textile.

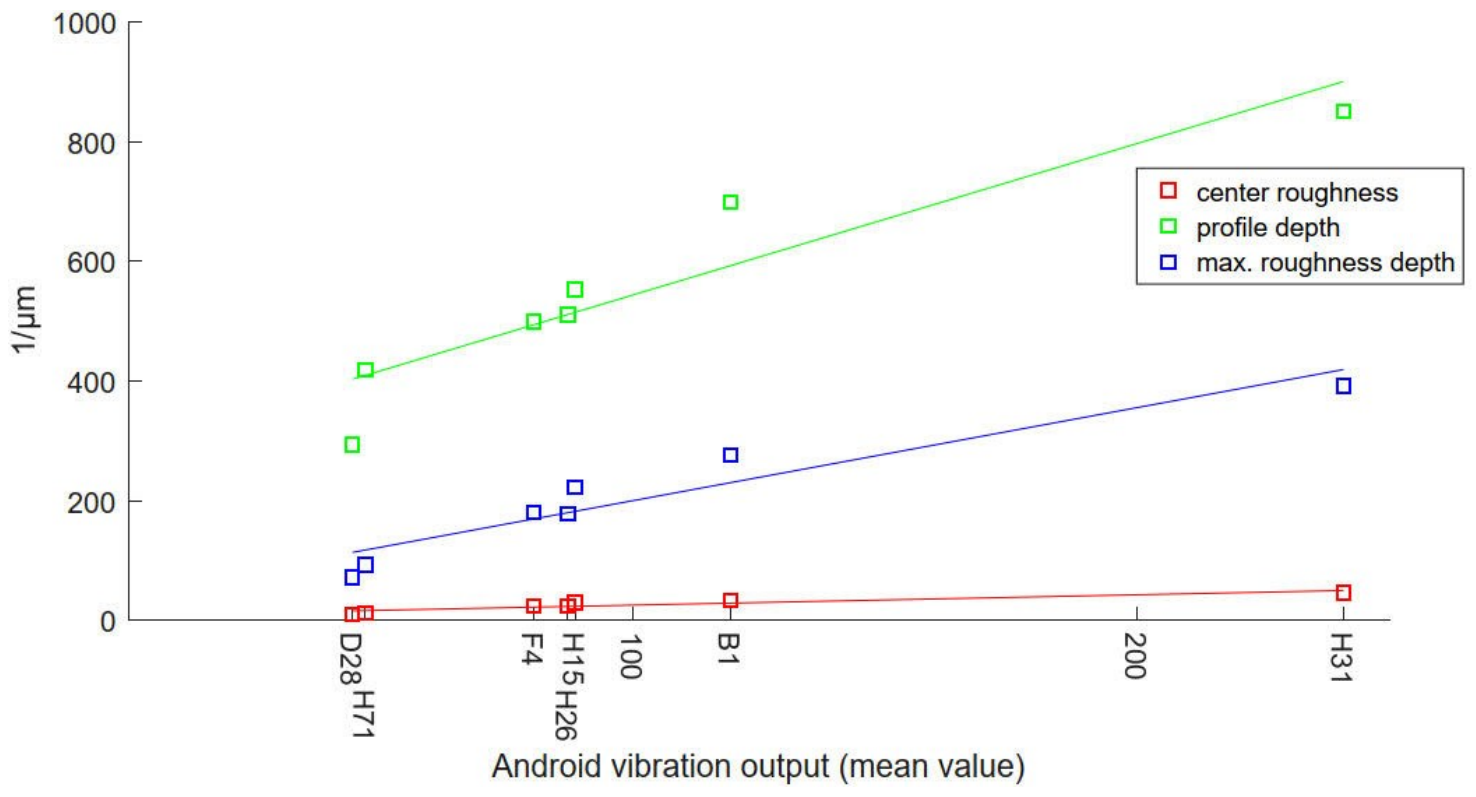

Fig. 7: Correlation between optical roughness and vibration output

However, the smartphone's surface and the haptics engine's properties proved to be limiting. The hardware's shortcomings were particularly evident on very smooth and heavily textured surfaces. The motor's non-centered placement also falsifies the user's sensation.

Several test persons registered a heat development of the display as annoying when brushing over the hard glass surface. The heat development should therefore be taken into account in future developments. Sweat on the fingers also affects the sensation. Other surfaces or a cover could help. 
Finally, subjects were asked whether this application would influence their online shopping behavior. Despite the limitations, five replied, such a device would probably support their shopping decision, with three more being undecided.

\section{Acknowledgements}

The authors would like to thank Kathrin Pietsch and Cornelia Rataj for their contributions towards the virtual representation of textile fabrics as well as Sebastian Artur Lehmann and Stefan Große for their work on the Android application.

The IGF proposal 19479 BG of the research association "Forschungskuratorium Textil e. V." was funded via the AiF within the scope of the "Industrial Collective Research" (IGF) by the German Federal Ministry for Economic Affairs and Energy (BMWi).

\section{References}

[1] N. Magnenat-Thalmann, P. Volino, U. Bonanni, I. R. Summers, M. Bergamasco, F. Salsedo and F.-E. Wolter. 2007. From physics-based simulation to the touching of textiles: the HAPTEX project. The International Journal of Virtual Reality 6, 3, 35-44.

[2] Tanvas Inc. Sample Applications - Tanvas. 2021. Retrieved May 7, 2021 from https://tanvas.co/sampleapplications.

[3] Haptische Displays: Die Zukunft fühlbarer Tasten auf flachen Bildschirmen. Retrieved April 20, 2020 from https://www.trendsderzukunft.de/haptische-displays-die-zukunft-fuehlbarer-tasten-auf-flachen-bildschirmen/.

[4] D. Zühlke. 2012. Nutzergerechte Entwicklung von Mensch-Maschine-Systemen: Useware-Engineering für technische Systeme (2nd ed.) Springer-Verlag, Berlin and Heidelberg.

[5] M. Grunwald. 2008. Human Haptic Perception: Basics and Applications. Birkhäuser Verlag, Basel, Berlin and Boston.

[6] G. A. Gescheider. 1997. Psychophysics: The Fundamentals (3. ed.). Lawrence Erlbaum Associates, New Jersey and London.

[7] T. A. Kern and M. Matysek. Entwicklung haptischer Geräte: Ein Einstieg für Ingenieure (7. ed.). SpringerVerlag, Berlin and Heidelberg.

[8] K. O. Johnson and J. R. Phillips. 1981. Tactile spatial-resolution. I. Two-point discrimination, gap detection, grating resolution, and letter recognition. Journal of Physiology 46, 6, 1171-1191.

[9] K. A. Kaczmarek and P. Bach-y-Rita. 1995. Tactile Displays. In W. Barfield and T. A. Furness III (Eds.). Virtual environments and advanced interface design. Oxford University Press, New York, 349-414.

[10] Intuitive Surgical Inc., Intuitive | da Vinci Robotic Assisted Surgical System. 2019. Retrieved May 27, 2019 from https://www.intuitive.com/.

[11] V. G. Chouvardas, A. N. Miliou and M. K. Hatalis. 2008. Tactile displays: Overview and recent advances. Displays 29, 185-194. DOI: https://doi.org/10.1016/j.displa.2007.07.003.

[12] H. Böse, H. Ermert, A. Tunayar, G. Monkman, M. Baumann, W. Khaled, S. Reichling, O. T. Bruhns, H. Freimuth and S. Egersdörfer. 2004. A novel haptic sensor-actuator system for applications in virtual reality. Proc. 4th Internat Conf. EuroHaptics 2004, 88-93.

[13] G. Paschew. 2020. Intermodale Displays auf Basis intrinsisch aktiver Polymere, Technische Universität Dresden.

[14] D. Allerkamp, G. Böttcher, F.-E. Wolter, A. C. Brady, J. Qu and I. R. Summers. 2007. A vibrotactile approach to tactile rendering. The Visual Computer 23, 97-108. DOI: https://doi.org/10.1007/s00371-006-0031-5.

[15] Y. Ikei and M. Shiratori 2002. TextureExplorer: A Tactile and Force Display for Virtual Textures. In Proceedings of the 10th Symposium on Haptic Interfaces for Virtual Environment and Teleoperator Systems, Washington.

[16] G.-H. Yang, K.-U. Kyung, M. A. Srinivasan and D.-S. Kwon. 2006. Quantitative tactile display device with pinarray type tactile feedback and thermal feedback. In Proceedings 2006 IEEE International Conference on Robotics and Automation ICRA 2006.

[17] K.-U. Kyung, M. Ahn, D.-S. Kwon and M. A. Srinivasan, 2005. A compact broadband tactile display and its effectiveness in the display of tactile form. In First Joint Eurohaptics Conference and Symposium on Haptic Interfaces for Virtual Environment and Teleoperator Systems. World Haptics Conference.

[18] E. Freeman, R. Anderson, J. Williamson, G. Wilson and S. A. Brewster. 2017. Textured Surfaces for Ultrasound Haptic Displays. In Proceedings of the 19th ACM International Conference on Multimodal Interaction, New York, USA. 
[19] O. Bau, I. Poupyrev, A. Israr und C. Harrison. 2010. Teslatouch: Electrovibration for Touch Surfaces. In UIST'10: Proceedings of the 23nd annual ACM symposium on User interface software and technology, New York, USA.

[20] M. E. Altinsoy and S. Merchel. 2012. Electrotactile feedback for handheld devices with touch screen and simulation of roughness. IEEE Transactions on Haptics 5, 6-13. DOI: https://doi.org/10.1109/TOH.2011.56.

[21] Texas Instruments. 2013. Haptics: Solutions for ERM and LRA Actuators. Retrieved June 6, 2019 from http://www.ti.com/lit/ml/sszb151/sszb151.pdf.

[22] Applelnsider. 2016. Inside the iPhone 7: Apple's Taptic Engine, explained. Retrieved Jung 6, 2019 from: https://appleinsider.com/articles/16/09/27/inside-the-iphone-7-apples-taptic-engine-explained.

[23] Sony Mobile Communications. 2018. Meet the Makers: Die Macher hinter der Unterhaltungsrevolution - Sony Mobile (Deutschland). Retrieved Jung 21, 2019 from https://blogs.sonymobile.com/de/2018/05/25/meetmakers-dynamic-vibration-system/.

[24] E. Steinbach, M. Strese, M. Eid, X. Liu, A. Bhardwaj, Q. Liu, M. Al-Ja'afreh, T. Mahmoodi, R. Hassen, A. E. Saddik and O. Holland. 2019. Haptic codecs for the tactile internet. Proceedings of the IEEE 107, 447-470. DOI https://doi.org/10.1109/JPROC.2018.2867835.

[25] Google Inc. 2019. Vibrator | Android Developers Retrieved July 17, 2019 from https://developer.android.com/reference/android/os/Vibrator.html.

[26] Google Inc. 2019. MotionEvent | Android Developers. Retrieved June 18, 2019 from https://developer.android.com/reference/android/view/MotionEvent.

[27] M. Jungmann. 2004. Entwicklung elektrostatischer Festkörperaktoren mit elastischen Dielektrika für den Einsatz in taktilen Anzeigefeldern. Dissertation Thesis, Technische Universität Darmstadt. 